\title{
Objetos de aprendizaje como recursos educativos en programas de alfabetización en información para una educación superior de posgrado competencial
}

\author{
Miguel Ángel Marzal \\ Javier Calzada Prado * \\ Eduardo Ruvalcaba Burgoa **
}

Artículo recibido:

22 de enero de 2014.

Artículo aceptado

30 de abril de 2014.

\section{RESUMEN}

El presente trabajo analiza la relevancia de la evaluación de contenidos digitales educativos en la adquisición de competencias en información por parte de los alumnos de posgrado dentro de programas de alfabetización en información. Se trata en primer término el consumo de información para adquirir conocimiento de los estudiantes de Posgrado en Ciencias Sociales; en seguida se estudian las características necesarias de los contenidos educativos digitales para determinar la calidad en el aprendizaje con el fin de definir la eficacia de los objetos de aprendizaje (OA) dentro de programas específicos de alfabetización en información. A continuación se expo-

* Ambos autores pertenecen a la Universidad Carlos III de Madrid, España. (mmarzal@ bib.uc3m.es); (fcalzada@bib.uc3m.es)

**El Colegio de México, A.C. México. eruvalcaba@colmex.mx

INVESTIGACIÓN BIBLIOTECOLÓGICA, Vol. 29, Núm.66, mayo/agosto, 2015, México, ISSN: 0187-358X. pp. 139-168 
ne la relevancia de la evaluación de contenidos digitales educativos como un medio demostrativo de la competencia en información y un instrumento para obtener conocimiento, por lo que se propone un modelo de criterios evaluativos del uso de objetos de aprendizaje, exponiendo una aplicación como medio para desarrollar programas de alfabetización en información basados en el uso competente de los objetos de aprendizaje.

Palabras clave: Objetos de aprendizaje; Alfabetización en información; Contenidos digitales educativos; Evaluación de contenidos digitales.

\section{Abstract}

Learning objects as a resource in information literacy in competency-based, post-graduate degree programs Miguel Ángel Marzal, Javier Calzada-Prado and Eduardo Ruvalcaba-Burgoa

By looking closely at the evaluation de digital contents of educational materials, this work examines information competencies within information literacy programs and their impact on the performance of postgraduate students. An initial examination of Social Sciences postgraduate student academic information consumption habits is made, followed by an analysis of the features of digital educational contents required to assess quality of learning and determine the efficiency of learning objects within specific information literacy programs. The paper then presents the impact of the assessment of digital educational contents as a means of demonstrating information literacy and as a tool for acquiring knowledge. The researchers also propose a model with evaluation criteria for assessing use of learning objects and the development of information literacy programs based on the competent use of learning objects.

Keywords: Learning Objects; Information Literacy; Educational Digital Contents; Educational Contents Assessment. 


\section{INTRODUCCIÓN}

T a naturaleza académica de la alfabetización educativa tiene distintas verLtientes según sea el contexto formativo y su finalidad. Cuando aparece concebida como una especialidad académica, su objetivo se dirige a procurar competencias en información para el desempeño de una actividad investigadora (estudiosos de posgrado), así como profesional (documentalistas en centros de recursos, bibliotecas digitales y virtuales). Esta concepción se proyecta en el diseño de materias curriculares dentro de los estudios de grado o posgrado, con su propio propósito instructivo conformado por objetivos, metodología, estructura y objeto dentro del currículo.

La naturaleza académica de la alfabetización en información reconoce a las bibliotecas digitales educativas como el espacio idóneo para ejercitar las competencias en información. Calzada (2010) ha expuesto cómo el término de biblioteca digital con el adjetivo "educativa" inició su visibilidad entre los especialistas hacia 2001, a partir de proyectos cooperativos para el desarrollo de bibliotecas digitales académicas entre el National Science Foundation estadounidense y el británico JISC (Joint Information Systems Committee), como el programa eLib (Electronic Libraries Programme). Uno de los valores propios de estas bibliotecas consiste en procesar contenidos y recursos aportados por los usuarios, así como estar tratados conforme a los recursos y estándares educativos, por lo que una de sus funciones debe ser el desarrollo, tratamiento y difusión de repositorios digitales, cuyos principales elementos son los objetos digitales educativos y los objetos de aprendizaje (OA). Estas bibliotecas, además, deben guiarse por criterios de calidad asentados, entre otros, sobre la evaluación de contenidos y un acceso integrado para que el usuario perciba que la biblioteca es un elemento situado en un entorno virtual de aprendizaje, interactuación y organización de los contenidos conforme al interés y las necesidades de la comunidad educativa, para reutilizar y generar los recursos por parte de los docentes (Tarango y Marzal, 2011).

Los anteriores factores, unidos al hecho de que la instauración de los nuevos modelos educativos competenciales se esté produciendo en el segmento superior de los estudios (en la universidad y, dentro de ella, el posgrado), refrendan la necesidad de investigaciones y publicaciones en torno a los caracteres y propiedades que deben acompañar a los programas de alfabetización en información para docentes y discentes de posgrado, mediante el uso eficaz de materiales didácticos web idóneos, objetos digitales educativos y OA. A este cometido se orienta el presente trabajo. 


\section{USO DE LA INFORMACIÓN PARA EL CONOCIMIENTO EN EDUCACIÓN SUPERIOR}

\section{Información para el conocimiento entre estudiantes de posgrado}

La investigación se dirige a estudiantes de posgrado, con el fin de analizar el uso de la información para su conversión en conocimiento y observar las características y necesidades propias y diferenciadas de otros usuarios universitarios (Kohl-Frey, 2008), especialmente las referidas a la demanda de servicios más especializados y personalizados, la consulta de gran cantidad de fuentes de información, la búsqueda de recursos impresos y electrónicos en una red educativa de bibliotecas con catálogos colectivos y el acceso a recursos web.

Uno de los rasgos prototípicos de este tipo de estudiantes es su carácter heterogéneo en formación, motivación, expectativas o dedicación. Hallamos estudiantes recién egresados de una licenciatura sin experiencia laboral ni en cuestiones de investigación, pero con un notable nivel de habilidades tecnológicas; al tiempo que encontramos estudiantes de mayor edad que cuentan con experiencia laboral, no necesariamente en el área de estudio de su especialidad, y en muchos casos con ciertas habilidades en la investigación, aunque a veces desactualizadas en sus aspectos instrumentales. Estas circunstancias condicionan fuertemente la relación del estudiante de posgrado con la información para el conocimiento, y no deben desconocer los medios y modos de uso de las bibliotecas universitarias que ya son híbridas y, cada vez más, bibliotecas digitales (a veces virtuales).

Estudios específicos acreditan que un alto porcentaje de estudiantes de posgrado $(77.3 \%)$ considera la Internet como fuente principal de información, frente a un porcentaje menor $(22.3 \%)$ que considera a la biblioteca en el mismo sentido (Saiti y Prokopiadou, 2008), si bien esta proporción varía dependiendo del área de conocimiento y especialidad del estudiante. Esto no obsta para que la imagen de la biblioteca universitaria sea el centro fundamental para las fuentes y recursos de información entre la mayoría de estudiantes de posgrado exactamente en un 85.7 \% (Kayongo y Helm, 2010).

\section{Tipificación de estudiantes de posgrado, su espacio e instrumentos para el conocimiento}

La identificación de estudiantes universitarios bajo el epígrafe de posgrado es, sin duda, un ejercicio de simplificación para un análisis científico que requiere un refinamiento en su tipificación. La comunidad de posgrado de 
las universidades implica diferencias entre sus requerimientos de información (Barry, 1997) entre los estudiantes de másteres y doctorado, sobre todo en cuanto al manejo de buscadores y gestores de referencias bibliográficas (Harrington, 2009), pero son usos de escasa relevancia. De hecho, al enunciar ambos tipos de estudiantes sus más inmediatas y útiles competencias de información enuncian como prioritarias la elaboración canónica del trabajo académico, los modos de actualización de conocimientos en su especialidad y la pericia en el uso experto de fuentes de información (Hoffmann et al., 2008). Sin embargo, no es menos cierto que el consumo de información sea diferente según las áreas de conocimiento. A efectos de este trabajo nos circunscribiremos a los estudiantes de Posgrado en Ciencias Sociales.

Sin duda, una exigencia absolutamente esencial en el proceso de elaboración de una tesis doctoral es la actualización, en tanto que también lo es la profundidad argumentativa y epistemológica, visible en el distinto periodo de tiempo contemplado para la presentación y defensa de una tesis doctoral y una de fin de máster. No abunda la literatura científica respecto del comportamiento informativo de los estudiantes de doctorado, y cuando existe son análisis que se derivan de metodologías y áreas de conocimiento muy distintas, por lo que los resultados obtenidos difieren, aunque demuestran la especificidad que guardan las necesidades de información de los estudiantes. Esta tipificación y análisis diferenciado, además, se fundamenta en estudios y buenas prácticas de bibliotecas destinadas específicamente a atender a alumnos de posgrado, las que consideran sus necesidades en cuanto a servicios, recursos de información y, particularmente, los espacios adecuados para estudiar y realizar sus investigaciones ya se trate de bibliotecas auxiliares de un centro de posgrado especializado o de un espacio reservado para estos estudiantes en el espacio de una biblioteca universitaria. Estos lugares reclaman la estructura tecnológica que permita ofrecer servicios y recursos de información para dichos estudiantes (Sadler y Given, 2007).

Otro factor relevante lo constituyen las fuentes de información que consultan los especialistas en ciencias sociales, rasgo esencial de los hábitos informativos de los estudiantes de posgrado que ha sido materia de análisis en diversos estudios. Romanos de Tiratel (2000) presenta algunos de estos aspectos: uso intensivo de materiales actualizados, consulta a colegas y expertos, seguimiento de citas localizadas en revistas, uso importante de material publicado en su lengua materna y preferencia similar por libros y revistas. Respecto de esta última característica, el mismo estudio señala una tendencia hacia el uso de un mayor porcentaje de libros ( $47.1 \%$ ) que de revistas $(30.4 \%)$, tendencia que reafirman los resultados presentados en el informe Researchers' use of academic libraries and their services (Consortium of Uni- 
versity Research Libraries \& Research Information Network, 2007). Por su parte, el estudio de Saiti y Prokopiadou (2008) citado señala entre sus conclusiones que dicha selección puede ser atribuida principalmente a cuatro factores: 1) la disposición de información fiable y actualizada, 2) la facilidad de acceso a la información en casa, 3) la hora del día en que se puede obtener el material (tiempo del recurso), y 4) la velocidad de recuperación de información. Harrington (2009) destaca la preferencia de los alumnos de posgrado por el acceso electrónico a los recursos, pues si no encuentran respuestas a su necesidad informativa en las fuentes de información digital de la biblioteca acuden a buscadores genéricos de información web, obviando las fuentes impresas, lo que implica una disfunción para la biblioteca.

\section{CONTENIDOS Y RECURSOS EDUCATIVOS DIGITALES}

\section{Precisiones conceptuales}

Un proceso de enseñanza y aprendizaje utiliza como apoyo, dependiendo de la metodología propia de la función docente, materiales didácticos para transmitir contenidos educativos. Tales materiales se proyectan en dos maneras: el recurso educativo, entendido como cualquier material que, en un contexto educativo determinado, sea utilizado con una finalidad didáctica o para facilitar el desarrollo de las actividades formativas; y el medio didáctico, como cualquier material elaborado con la intención de facilitar los procesos de enseñanza y aprendizaje, ya que sirven para organizar y relacionar conceptos, orientar su aplicación, generar conocimiento y estimular el interés del educando para desarrollar en él destrezas, habilidades y competencias. La distinción es importante: los recursos educativos pueden ser utilizados en un contexto educativo, pero no han sido creados necesariamente para esta función y suelen tener un carácter compacto (por ejemplo, la prensa); los medios didácticos, sin embargo, son recursos educativos que tienen como propiedades inherentes una intencionalidad educativa y el propósito de un uso didácti$c o$, por lo que pueden adaptarse a una programación en unidades didácticas. En un programa de alfabetización informativa son particularmente relevantes los medios didácticos denominados recursos de información educativos, que recopilan y organizan una información hacia el conocimiento.

Los medios didácticos se soportan sobre materiales didácticos para la transmisión de contenidos educativos, donde el instrumento de difusión se hace muy importante, especialmente el digital. Surgen así "objetos de información en soporte digital”, muy distintos según su codificación (texto, audio, 
video, multimedia), almacenamiento (CD-ROM, DVD, etc.) y su funcionalidad (herramientas informáticas, portales, sitios web, webs institucionales, etc., todos ellos educativos). Para Gértrudix et al. (2007) los contenidos educativos digitales se refieren tanto al producto como a los procesos que los han motivado y permitido diseñar su reutilización, por lo que se refieren tanto a productos digitales a partir de un modelo constructivo (que los autores llaman bricosoft) hasta productos integrales presentados como paquetes. A partir de estas consideraciones aparece el concepto de recursos web o digitales educativos, por su soporte digital y sus contenidos educativos web.

\section{Tipos de recursos educativos digitales para conocer y saber en la Web: $R E A, O D E, O A$}

Los contenidos digitales educativos, pues, reclaman competencias propias para que la información contenida pueda transformarse en conocimiento y saber. Sus propiedades se presentan, se acceden y se usan de maneras diferentes según su entorno de aplicación, su modalidad y objetivos competenciales educativos, y su funcionalidad didáctica. Estas condicionantes rodean a los Recursos Educativos Abiertos (REA) u Open Educational Resources (OER) y los Objetos Digitales Educativos (ODE).

Los REA se refieren tanto a contenidos formativos como a herramientas e implementaciones (estándares, instrumentos, etc.) y forman parte tanto del movimiento de acceso abierto a la Ciencia, u Open Access, como del software de código abierto por el movimiento Open Source Software (OSS), y del contenido en general mediante el movimiento Open Content. La iniciativa del consorcio mundial Open CourseWare (OCW) es particularmente relevante para los REA, en especial respecto a los contenidos en forma de cursos, y unida necesariamente a la iniciativa para la distribución y acceso de REA a escala internacional del repositorio de metadatos o pasarela a contenidos educativos abiertos OERCommons, lanzado en 2007 por ISKME (Institute for the Study of Knowledge Management in Education).

En LOM-ES, los ODE se caracterizan por el tipo de información representada (textual, audiovisual, virtual, etc.) y por su aplicabilidad en diseños instructivos concretos, fenómenos que determinan niveles de granularidad para categorizar estos ODE dentro de una arquitectura modular de jerarquía creciente. El nivel de granularidad del ODE atiende a su estructura o composición, funcionalidad en el proceso educativo y cobertura curricular aproxima$d a$ de sus contenidos educativos. G. Bueno (2010) describe los cuatro niveles de los ODE: el nivel 1 (Objeto básico) integra aplicaciones informáticas y sus servicios, sistemas de representación de la información y el conocimiento, 
los media (fotografía, video, música, hipertexto, etc.) y los multimedia; nivel 2 (OA), un objeto que se caracteriza por ser el nivel más pequeño e incluir una función didáctica explícita (Diseño instruccional o Instructivo); nivel 3 (Secuencia Didáctica), estructurado por un conjunto determinado de objetos digitales de nivel 2, y excepcionalmente, de nivel 1, con actividades de aprendizaje y evaluación implícitas; nivel 4 (Programa de Formación), que puede cubrir un área de conocimiento completa de un nivel educativo determinado.

Los ODE, por lo demás, tienen como propiedades bien definidas las siguientes:

- La reutilización, en diferentes contextos educativos y en distintas unidades o módulos didácticos. Esta propiedad reclama su acceso abierto, en la dimensión económica, legal y técnica en código abierto, así como la accesibilidad, por requisitos tecnológicos simples y la usabilidad de sus contenidos.

- La interoperabilidad, con el objetivo de ser empleados en cualquier plataforma y entorno de aprendizaje distinto para el que fueron creados, independientemente del hardware, sistema operativo, navegador o programa que permita su utilización por parte del usuario. Esta propiedad se apoya en la portabilidad para el empaquetamiento y transferencia de contenidos entre sistemas y herramientas mediante estándares.

- La disponibilidad respecto a la búsqueda, localización y recuperación mediante buscadores en red, por lo que ha de ser digital, multimedia $e$ interactivo, para permitir su actualización, reduplicación y distribución.

- La finalidad didáctica, para garantizar que el proceso de aprendizaje es efectivo mediante la calidad de los contenidos y el seguimiento del progreso competencial del educando, por lo que deben ser explícitos los objetivos didácticos y competenciales a través de instrucciones y criterios de evaluación.

Respecto a los OA, su noción se debe a Merrill (1983), en la década de 1970, al hablar de los objetos de conocimiento, como el uso de componentes digitales en la formación y educación. El concepto (Learning Objects) apuntó en la década de 1990 por las aportaciones de Hodgings (2002) y su metáfora de las piezas de Lego para explicar la construcción de materiales formativos con base en pequeños componentes, por un lado, y por el otro a Metros y Bennett (2002), quienes relacionan el concepto con la Programación Orientada a Objetos (POO), que 
establece la reutilización del código para el desarrollo de software como elemento definitorio fundamental hasta aparecer en 1998 el término Reusable Learning Objects para referirse a cada tema y configurar una lección de aprendizaje. A este elemento básico de definición se unieron la enunciación de objetivos de aprendizaje, de características educativas en forma de metadatos y de un componente de evaluación. La metáfora del Lego se refiere a la disposición de una serie de componentes (unidades de contenido) que, combinados a la manera de piezas, sirvieran para diseñar experiencias de aprendizaje adaptadas a las diferentes necesidades de los educandos; esto no pareció muy afortunado por lo que se complementó con la metáfora del átomo, ya que no todos los elementos que componen un objeto de aprendizaje pueden combinarse con otros, pues la combinación depende de elementos como el contexto de aplicación.

Si bien existe un gran número de "herramientas de autoría de contenidos" (authoring tools) para crear contenidos digitales para e-learning, según se muestra en el directorio creado por Jane Hart y disponible en http://www. c4lpt.co.uk/Directory/Tools/authoring.html, sólo dos se han proyectado en el ámbito educativo: CourseGenie es una aplicación que funciona como plugin de Microsoft Word y que permite generar OA en HMTL y paquetes SCORM e IMS a partir de textos; Reload (Reusable Learning Object Authoring and Delivery) es una aplicación opensource para la creación de paquetes de contenido desarrollada gracias a la financiación de la entidad británica JISC (Joint Information Systems Committee, http://www.jisc.ac.uk/).

Así, por su naturaleza conceptual más precisa, su función didáctica más claramente explícita, así como por su carácter modular que puede determinar la Secuencia Didáctica y el Programa de Formación, los OA son los recursos digitales que hemos seleccionado y los que vamos a analizar para su eficaz aplicación en un programa de alfabetización en información.

\section{LOS OA PARA PROGRAMAS DE ALFABETIZACIÓN EN INFORMACIÓN}

\section{Una definición: una convención necesaria para su aplicación. Sus propiedades}

La dificultad de alcanzar una definición consensuada para los OA se ha convertido en uno de los principales problemas para su avance, y se basa en la heterogeneidad de los contenidos educativos. Entre las numerosas definiciones cabe distinguir dos tendencias: 
- Aquéllas que establecen que un OA puede ser cualquier objeto con utilidad educativa (defendida principalmente por el ámbito de las tecnologías educativas). En esta tendencia, McGreal (2004) acrisoló cinco categorías para definir los $\mathrm{OA}$ : a) cualquier cosa y todas las cosas; b) cualquier cosa digital, con o sin finalidad educativa; c) cualquier cosa para el aprendizaje; d) sólo objetos digitales con una intencionalidad educativa; e) sólo objetos digitales cuya intencionalidad educativa haya sido marcada o expresada formalmente. Sólo las tres primeras categorías entran en esta primera tendencia. En la primera categoría se enmarca la definición de LTSC (Learning Technology Standards Committee) del IEEE, "es una entidad, digital o no, que puede ser usada o referida durante un proceso de aprendizaje basado en la tecnología" (LTSC, 2002: s. pág.); en la segunda categoría destaca Wiley (2000), que sólo integra los recursos en formato digital; en la tercera categoría se acepta el uso didáctico pero no la finalidad didáctica, según expresan en Doorten et al. (2004).

- Aquéllas que defienden que un OA debe poseer características concretas y bien asentadas en el ámbito educativo y documental. Para Metros (2005), un objeto de aprendizaje debe contener, al menos, los siguientes elementos: un objetivo de aprendizaje, una actividad práctica y una evaluación. Las dos últimas categorías de McGreal se inscriben en esta tendencia: la cuarta categoría que introduce la finalidad didáctica es defendida por Koper (2003) y Polsani (2003), la quinta categoría sería una prolongación perfectible de la cuarta, pues los objetos digitales con un propósito educativo formal deben llevar explícito este objetivo (mediante metadatos), de modo que los OA estarían compuestos por tres elementos: contenido+objetivos+metadatos. Así, se llega a la definición de Barritt y Alderman: "un objeto de aprendizaje es una colección independiente de elementos de contenido y medios, un enfoque de aprendizaje (interactividad, arquitectura del aprendizaje, contexto) y metadatos (empleados para el almacenamiento y la búsqueda)" (2004: 7-8), estos autores especifican claramente que los metadatos son parte de la definición.

Respecto a las propiedades de los OA se han resumido con el acrónimo RAID (Reusability, Accesibility, Interoperability y Durability), a lo que se unen la finalidad didáctica o el diseño instruccional, el carácter digital y multimedia, y la interactividad.

Por su naturaleza digital los OA deben expresarse con distintos códigos semióticos (texto, imagen, audio, video), aportando necesariamente la mul- 
timedialidad y la interactividad mediante actividades, simulaciones, cuestionarios, diagramas, experimentos, etc. Si bien son propiedades comunes a otros materiales didácticos, es la finalidad didáctica la que hace únicos a los $\mathrm{OA}$, una finalidad que se hace explícita en el diseño instructivo. Un requisito fundamental del diseño instructivo es la reutilización, por lo que los OA incorporan algunas condiciones necesarias: ser modulares, es decir, que se puedan descomponer en unidades de pequeño tamaño; ser independientes y autónomos del resto de objetos que los rodean; estar descontextualizados de materias o currículos académicos; ser durables en el tiempo; indicar algunos de los posibles contextos de uso y facilitar el proceso posterior de implementación; contar con características identificativas (metadatos); ser recuperables y localizables a partir de sus principales características descritas conforme a metadatos.

La reutilización, además, implica la granularidad o nivel de agregación y se refiere al tamaño, detalle, complejidad o alcance de aquello que es considerado como un recurso por sí mismo y fundamento de la arquitectura modular, precisamente la modularidad facilita la adaptabilidad y reutilización de los objetos que componen una unidad educativa, además de favorecer la posibilidad de reelaborar los contenidos. Los OA deben responder, por último, a otras dos propiedades: la interoperabilidad, para que puedan ser independientes del hardware, sistema operativo, navegador o programa que permita su utilización por parte del usuario, y la disponibilidad, por los metadatos, de modo que éstos se deberían almacenar y organizar convenientemente en sistemas de acceso en línea, y que estos sistemas faciliten su búsqueda, localización y recuperación conforme a múltiples criterios.

\section{Los $O A$ en programas de alfabetización en información}

Según muestra la literatura científica y profesional, los estudiantes universitarios y, por ende, los estudiantes de posgrado, manifiestan deficiencias en el manejo de la información, pero también en la edición de trabajos académicos, tal como demostraba el trabajo de campo de Harrington (2009), donde se recogía que un 51 \% de los estudiantes de Posgrado en Psicología reconocía necesitar esta formación en información, aun cuando el autor constataba que los estudiantes desconocían muchos instrumentos de procesamiento de información y, por tanto, también sus beneficios. Subvenir estas deficiencias recae en la naturaleza de la alfabetización en información como especialidad que faculta en las competencias en información.

Parece imprescindible incorporar un programa competencial de alfabetización en información durante la formación de los estudiantes de posgra- 
do, pero de manera que se asocien las actividades de alfabetización en información con los proyectos de investigación de los estudiantes. Los cursos de competencias informativas servirían para resolver cuestiones específicas en sus trabajos de investigación, por cuanto al tiempo que adquieren las competencias el programa pivota sobre ejercicios y actividades sobre su tópico de investigación (Newby, 2011), por lo que parece idóneo que estos cursos competenciales se impartan al comienzo o durante el proceso de investigación en el posgrado. En un interesante estudio, Catalano (2010) defendía que, estudiadas las necesidades informativas y las competencias necesarias, la formación de los estudiantes de posgrado superaba con creces la instrucción bibliográfica y bibliotecaria, como también la formación de usuarios. Sin embargo, existe una importante disfunción: en una encuesta llevada a cabo para conocer la actividad formadora de bibliotecarios, Mestre et al. (2011) demostraban que más de la mitad de ellos jamás había editado recursos didácticos web y aún menos $\mathrm{OA}$, si bien tienen experiencia en la evaluación de recursos de información, lo que los faculta para evaluar, con criterios objetivos, los OA susceptibles de incorporarse a cursos de alfabetización en información.

Para Dewald et al. (2000) son tres las vías para la realización de dicha formación en el entorno virtual: a) cursos ALFIN con reconocimiento de créditos, b) formación ALFIN integrada en cursos del currículo y c) tutoriales web sobre ALFIN. Holmes (2003) ha descrito la experiencia de la Universidad de Washington en el diseño de un conjunto de tutoriales que servían a los bibliotecarios en la enseñanza de la alfabetización en información. Los tutoriales UWill (University of Washington Information Literacy Learning) son plantillas de páginas web que contienen elementos esenciales asociados con la producción, distribución, recuperación y evaluación de información, y están basadas en las Normas de la ACRL. Similar experiencia se encuentra en la Biblioteca del Congresista Frank J. Guarini de la New Jersey City University (http://www.njcu.edu/Guarini/Instructions/ILTutorial/ILTutorial. $\mathrm{htm})$. En las tres modalidades, los contenidos ALFIN deben incorporar el aprendizaje activo e instrumentos de evaluación que permitan al estudiante determinar el grado de aprendizaje alcanzado y mejorar su formación. Es precisamente este aspecto el que presenta la mayor debilidad de los tutoriales para alfabetización en información: presentan un "consumo" pasivo para un e-learning tradicional y tienen un sesgo declarado hacia la formación de usuarios en línea, por lo que son precisos criterios e indicadores para asegurar su eficacia en conocer (Somoza y Fernández, 2011).

Por el contrario, los OA deben ser actividades prácticas en las que el usuario pueda controlar su aprendizaje. Convertidos en recursos didácticos, los OA deben acogerse a un determinado diseño pedagógico, pero además a una 
metodología docente concreta y a estrategias de aprendizaje dirigidas a las competencias. Los OA, por su granularidad, deberían diseñarse en cuatro capas: nivel 1 de agregación más simple (un texto, una imagen, un audio); nivel 2, un "tema"; nivel 3, una unidad didáctica; nivel 4, un módulo.

La naturaleza intrínseca de la alfabetización en información, pues, aconseja que sus programas pivoten sobre OA por su diseño instructivo. El diseño instructivo procura presentarle al educando los contenidos de forma que su experiencia de aprendizaje sea óptima, por lo que según Moreno y Bailly-Baillière (2002) debe aportar un enfoque orientado al diseño respecto a los objetivos planteados; un método de instrucción contextual, que tiene en cuenta las condiciones del aprendizaje; un método de instrucción modular, para la identificación de los objetivos propuestos, estrategias de enseñanza según el tipo de contenidos, secuenciación de actividades y evaluación; un método posibilista, para impulsar las posibilidades de alcanzar los objetivos. El diseño instructivo debe tener elementos de efectividad: planificación para el conocimiento y condiciones previos y necesarios en el proceso de enseñanza y aprendizaje; objetivos generales y específicos de aprendizaje; competencias generales para ser adquiridas; tipos de conocimiento programados (declarativo, procedimental, condicional, etc.); procesos cognitivos implicados (reconocimiento, recuerdo, aplicación, juicio crítico, etc.); método/s de aprendizaje y evaluación utilizado/s y criterios de evaluación planificados.

Como "materia", la programación de la alfabetización en información debe adoptar por tanto el diseño instruccional de los OA. Parece conveniente que el programa deba incluir seis elementos instructivos: guía de uso, con los objetivos competenciales que se busca conseguir y los principios teóricos necesarios para entender el módulo; ejemplos ilustrativos, para que los principios teóricos sean más eficazmente asumidos y transformados en habilidades; herramientas, software, para desarrollar la práctica de cada módulo del programa; material de apoyo, para que el alumno comprenda la utilidad de la aplicación; uso didáctico, esto es, la "práctica" del módulo, que acreditará la consecución de las competencias contempladas, y autoevaluación, seguimiento del propio progreso competencial. Más aún, cuando se contempla la programación de la alfabetización en información con un diseño modular y escalable, precisamente uno de estos módulos debe referirse a la Norma 5 de ANZIIL, destinada a la competencia en escritura digital, por uso experto de los modos de "escribir" en red, así como en la edición ética de los contenidos en OA, propios de cada alumno (Marzal y Parra, 2010). Por su parte, el Modelo ADDIE, desarrollado por la Universidad de Florida, propone una muy interesante categorización de los componentes educativos de los OA que contempla tres categorías, cada una con su progreso competencial: Fases, que incluye, en niveles de progreso, 
el análisis del proceso de aprendizaje, el diseño de aprendizaje, el desarrollo del proceso, la implementación del proceso con sus posibles aplicaciones y la evaluación; Tareas y Resultados, diferenciados según la secuencia de fases indicadas.

En este contexto, parece muy apropiada la propuesta de módulos de aprendizaje a partir de OA que publican Martínez Naharro et al. (2007), sobre todo a través de los objetos de acoplamiento. En definición de estos autores, se trata de objetos digitales que no cumplen completamente los requisitos para ser un objeto de aprendizaje pero no tienen sentido en sí mismos, sino al complementar a un OA, especialmente porque lo contextualizan y son el pilar idóneo para inscribir el OA en un módulo de aprendizaje. Los objetos de acoplamiento se convertirían en los vehículos apropiados para soportar, en un módulo de aprendizaje, la guía introductoria, las actividades prácticas, la evaluación y los resultados competenciales obtenidos dentro de un OA. Finalmente, desde esta perspectiva programática, los OA hallan una plusvalía para su efectividad mediante su gestión dentro de repositorios digitales específicos, los ROA. McGreal (2008) apuntó la tipificación de repositorios de OA como Repositorios tipo 1, que almacenan contenidos y presentan un número limitado de enlaces a contenidos externos; Repositorios tipo 2, que referencian contenidos externos, y Repositorios tipo 3, que albergan tanto contenidos como vínculos.

\section{LA EVALUACIÓN DE LOS OA EN PROGRAMAS DE ALFABETIZACIÓN EN INFORMACIÓN}

\section{La Evaluación en los $O A$}

En tanto que objetos digitales, los OA están sometidos a evaluación a partir de criterios procedentes de la accesibilidad y usabilidad de los recursos digitales. Si bien la accesibilidad hace referencia explícita a aspectos infraestructurales, que escapan a nuestro estudio, la usabilidad está definida en la ISO/IEC 9241 (1998), en su undécima parte, Guidance for usability, como el grado con que un determinado producto, en un contexto de uso específico, permite al usuario alcanzar sus objetivos con eficacia, eficiencia y satisfacción mediante la claridad, la capacidad de discriminación, la concisión, la coherencia, la identificabilidad, la legibilidad y la comprensibilidad.

Los OA son, sin embargo, recursos digitales educativos, por lo que a los anteriores criterios deben incluir otros como la percepción, la formación de conceptos, el almacenamiento en la memoria, la recuperación de la memoria 
y la contrastación de lo percibido con la memoria. Surgen, pues, trabajos sobre la usabilidad de los recursos educativos digitales, debidos a Nokelainen, Reeves, Squires y Preece, Quinn, Albion, entre otros, a partir de los que se han ido definiendo modelos de criterios de evaluación de estos recursos, entre los que podemos destacar los modelos de Reeves, Nokelainen, Zaharías y Marzal-Calzada-Vianello. A su vez, se han ido diseñando herramientas para la evaluación de estos recursos, como LORI, SREB-SCORE, HEODAR, EVALUAREED, entre otras (Pinto, Gómez y Fernández, 2012).

Por lo que llevamos argumentado, los OA, como un recurso educativo para programas de alfabetización en información, precisan de un modelo de evaluación específico basado en competencias. A este respecto es muy útil la reflexión que hacen Hernández, Silva y Velásquez (2012), para quienes la evaluación descansa sobre la calidad derivada del grado de cumplimiento de un conjunto de requisitos referidos a la funcionalidad y desarrollo del OA tecnológicamente, la usabilidad respecto a la interacción hombre-máquina y el grado de utilidad respecto a la consecución de los objetivos didácticos. Evaluar la calidad de los OA está determinando su uso eficaz en un contexto educativo específico, un módulo de aprendizaje, un curso y una secuencia didáctica. La evaluación de los OA, además, tiene otro fundamento: si la aplicación de los criterios evaluativos conforme a un modelo determinado se practicó sobre el OA en tanto que objeto digital, como acreditación de su proceso de edición, o respecto a la efectividad de sus contenidos digitales educativos en un proceso de enseñanza y aprendizaje, en tanto que producto didáctico.

Aun cuando la calidad siempre pivota sobre una percepción, esto es, sobre una valoración subjetiva, el proceso de evaluación, especialmente sobre el OA y sus características bien determinadas, debe acogerse a principios objetivos, con el fin de que los resultados evaluativos sean significativos tanto para el usuario como para el sistema que gestiona el sistema evaluador. La calidad primero y la evaluación después deben estar sometidas a parámetros de normalización para su tratamiento estadístico objetivo, de modo que existan cuestionarios que identifiquen la calidad, según derivados de indicadores.

Los considerandos anteriores han hecho que la calidad de los OA y su evaluación busquen un modelo de estándares que permitan diseñar herramientas de evaluación específicas. Pinto, Gómez y Fernández (2012) han presentado una interesante categorización para medir la siempre subjetiva calidad de los OA: calidad intrínseca (rigor, integridad, objetividad, precisión), calidad contextual (relevancia, valor añadido, actualidad, utilidad, adecuación), calidad representacional y calidad del acceso. Los instrumentos de evaluación, por su parte, se nutren de una lista de verificación a partir de modelos de criterios evaluativos, que se traducen en una plantilla para cuestionarios y luego en 
un método de análisis cuantitativo-cualitativo para procesar los datos de los cuestionarios, conforme al diseño de indicadores.

El proceso evaluativo apuntado es sumamente importante y práctico para su aplicación en los OA, en tanto que éste es un recurso en franca expansión entre los modelos educativos competenciales. Sin embargo, existe una dimensión realmente importante de los OA y aún más desde la perspectiva de este trabajo: ¿cómo evalúa el usuario-educando el OA en tanto que recurso educativo y qué competencias puede desarrollar éste?

\section{Evaluación de los $O A$ para un programa de alfabetización en información}

Los estudios en torno a los mecanismos de cognición en el procesamiento de información que hacen los seres humanos señalan como principales elementos de su transformación en conocimiento la captación selectiva, distribuida y sostenida en la usabilidad de los recursos electrónicos, la fidelización del recurso para la asimilación eficaz de su contenido y la capacidad alfabetizadora del recurso. Desde esta óptica, parece adecuado que el modelo de evaluación deba pretender hacer una medición del consumo de información educativa para luego hacer el diagnóstico de deficiencias competenciales, y por tanto medir el nivel de adquisición de destrezas en cuanto al uso de los aspectos tecnológicos; el nivel de adquisición de habilidades en la gestión de contenidos y el nivel de adquisición de competencias para la organización y representación de conocimientos en web, mediante la edición de conocimientos.

La evaluación, en todo caso, se apoyará en instrumentos de medición del impacto con finalidades diferenciadas; cuestionarios para valorar y percibir; indicadores para procesar los valores, los que para programas competenciales se pueden categorizar en: indicadores de diagnóstico, para evaluar deficiencias; indicadores de seguimiento, para evaluar el proceso; e indicadores de resultados, para verificar el cumplimiento de los objetivos y beneficios. Además, el sistema de evaluación se nutre de categorías para interpretar los datos y de procedimientos que consisten en los métodos de aplicación. Es bastante común que se utilicen indicadores cuantitativos para los programas de alfabetización en información en unidades de información, siempre en función del uso, cuando parece necesario que deban complementarse con "métodos cualitativos" debido a su mayor eficacia para evaluar actitudes, valoraciones y motivaciones (Viñas, 2004). 
Parece adecuado aportar ahora una experiencia destinada a evaluar los mecanismos de cognición arriba apuntados, estructurados a partir de requisitos competenciales que pueda proporcionar un OA y así medir la eficacia del aprendizaje conforme a objetivos competenciales. El objetivo, pues, será generar una plantilla definida de criterios evaluativos competenciales como herramienta para los alumnos con respecto al uso de los OA.

\section{Metodología}

La herramienta se plantea como un cuestionario atendiendo a criterios de evaluación descriptiva y predictiva e incluye una evaluación basada en conducta, otra basada en opinión y otra en usabilidad. Conforme al planteamiento de este trabajo, el universo estadístico objeto de estudio debe estar formado por estudiantes de Posgrado en Ciencias Sociales, a quienes se ofrece un $\mathrm{OA}$ seleccionado. Los métodos propuestos se explican a continuación.

\section{Modelo evaluativo}

El modelo evaluativo de los OA va a obviar, por el momento y en este caso, su procesamiento mediante un conjunto de indicadores, cuya complejidad en cuanto a su definición, etiqueta semántica y de finalidad, así como su tratamiento estadístico merecen un proyecto de investigación específico. La experiencia evaluativa se sostendrá, por tanto, sobre dos ejes: una tabla de categorías evaluativas como fundamento para el ulterior análisis de resultados y un cuestionario, que derivará necesariamente de la tabla, como elemento suministrador de los datos para el análisis.

La tabla de categorías evaluativas está alejada de los principios y caracteres de los instrumentos de evaluación de los OA existentes, que los procesan y evalúan en tanto que objetos digitales y no, según ya se ha argumentado, en tanto que recurso educativo y de formación en competencias informativas. Este sesgo nos ha llevado a formularlo a partir de los criterios y modelos evaluativos más acordes, especialmente los editados por Marzal, Calzada y Vianello en 2008; Pinto, Gómez y Fernández en 2012 y Hernández, Silva y Velásquez también en 2012.

Las categorías de la tabla, que recoge, redefine, funde o desarrolla los elementos evaluativos de los tres modelos indicados, se formulan a partir de los caracteres y las propiedades que se reconocen a los OA, por cuanto la experiencia evaluativa quiere asentarse sobre los beneficios de los OA en pro- 
gramas competenciales, si bien se añade espacio propio a la usabilidad como fundamento de la reutilización, y la propiedad intelectual, por haber sido ésta uno de los elementos más controvertidos en el desarrollo de los OA. La tabla quedaría diseñada de la manera siguiente:

Tabla 1. Categorías evaluativas para los OA

\begin{tabular}{|c|c|}
\hline Elemento de evaluación & Aspectos de evaluación \\
\hline Reutilización & $\begin{array}{l}\text { - Modularidad } \\
\text { - Autonomía del OA respecto al diseño curricular de disciplinas } \\
\text { - Granularidad } \\
\text { - Información para transformar, reescribir y reeditar el OA } \\
\text { - Requisitos de software bien identificados y sin costo } \\
\text { - Contenidos del OA sin dependencia exterior del propio OA }\end{array}$ \\
\hline Accesibilidad & $\begin{array}{l}\text { - Parte de un repositorio, con su sistema de almacenamiento y recuperación } \\
\text { - Velocidad en la ejecución de las distintas partes del proceso de uso del OA } \\
\text { - Mapa de navegación amigable por los enlaces del OA } \\
\text { - Auxiliaridad por existencia de normas de uso } \\
\text { - No requiere hardware, software, sistema operativo o navegador específicos } \\
\text { - Cumple los requisitos WAl (http://www.w3.org/WAI/) }\end{array}$ \\
\hline Interoperabilidad & $\begin{array}{l}\text { - Metadatos educativos según estándares, especificaciones o perfiles de } \\
\text { aplicación reconocidos } \\
\text { - Edición por herramientas interoperables }\end{array}$ \\
\hline Durabilidad & $\begin{array}{l}\text { - Estabilidad en el tiempo por gestión y mantenimiento } \\
\text { - Hipervínculos controlados en su funcionamiento y actualidad } \\
\text { - Dinamicidad de enlaces estructurada }\end{array}$ \\
\hline Multimedialidad & $\begin{array}{l}\text { - Contenido multimedia } \\
\text { - Recursos con múltiples estilos (audio, video, texto) } \\
\text { - Elementos gráficos y virtuales con propósito educativo } \\
\text { - Multisecuencialidad }\end{array}$ \\
\hline Interactividad & $\begin{array}{l}\text { - Actividades suficientes } \\
\text { - Respuestas correctas en breve tiempo } \\
\text { - Respuestas motivadoras e interactivas } \\
\text { - Multitarea }\end{array}$ \\
\hline Usabilidad & $\begin{array}{l}\text { - Extensibilidad por relación con otros OA o materiales web educativos } \\
\text { - Uso del OA sin entrenamiento previo } \\
\text { - Coherencia estilística, con interfaz legible, visible, intuitivo } \\
\text { - Elementos y signos-íconos de navegación metódica y sistemáticamente } \\
\text { estructurados y organizados } \\
\text { - Fiabilidad } \\
\text { - Amigabilidad } \\
\text { - Visualización con el menor desplazamiento }\end{array}$ \\
\hline Diseño instructivo & $\begin{array}{l}\text { - Información vigente, actual, sin errores, adaptada al educando } \\
\text { - Objetivos competenciales bien definidos } \\
\text { - Programación bien diferenciada de estos objetivos respecto a aprendizaje, } \\
\text { actividades y evaluación } \\
\text { - Propuesta de actividades conforme a los objetivos competenciales } \\
\text { - Contenidos y materiales educativos web del OA organizados conforme a } \\
\text { los objetivos competenciales }\end{array}$ \\
\hline
\end{tabular}




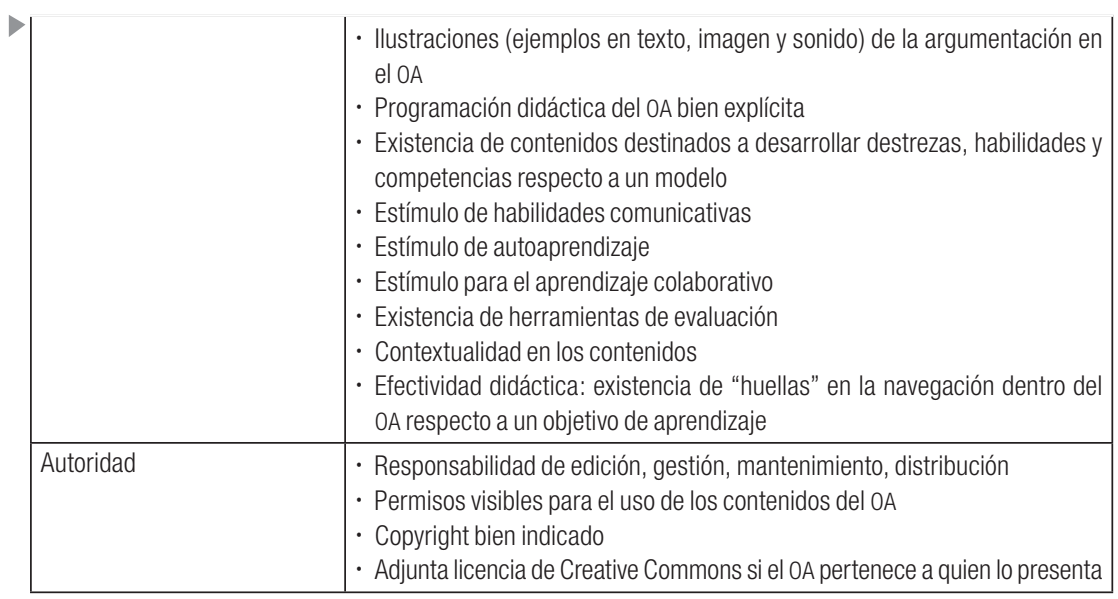

Fuente: elaboración propia

A partir de esta tabla de categorías evaluativas, se elabora un cuestionario para que los alumnos sometan el OA a evaluación; la experiencia tiene dos fases. En una primera el OA es sometido a los alumnos de forma libre para que lo evalúen y luego el mismo OA es evaluado por los alumnos siguiendo la tabla. El propósito principal de este procedimiento es buscar una metodología propia para poder elaborar recomendaciones que permitan un diseño y aplicación eficaces de los OA en programas competenciales.

\section{Selección de grupo-meta}

El universo estadístico seleccionado engloba a estudiantes de Posgrado en Ciencias Sociales, especialmente de los programas de maestría y doctorado en demografía, población y estudios urbanos que se imparten en el Colegio de México. Ambos programas de estudio forman parte del Programa Nacional de Posgrados de Calidad (PNPC) del Consejo Nacional de Ciencia y Tecnología (CONACYT).

Otros caracter definitorio es que se trata de estudiantes de reciente ingreso y que, en su mayoría, son personas que tienen una edad aproximada entre los 25 y los 35 años. Por pertenecer al PNPC, el CONACYT les brinda apoyo a través de una beca para que todos los alumnos dediquen tiempo completo a sus estudios, lo que significa que durante el periodo de estudios no pueden trabajar, dar clases o realizar alguna actividad que les reporte algún ingreso económico. En el primer semestre de su programa estudiaron como asignatura obligatoria un curso de investigación documental que imparte el personal académico de la Biblioteca Daniel Cosío Villegas, en donde se abor- 
dan los tópicos de la alfabetización en información. Entre estos contenidos se encuentra el estilo bibliográfico APA, cuyo aprendizaje complementan con el uso del gestor bibliográfico RefWorks. Al finalizar el curso, que se ha desarrollado entre agosto y octubre de 2013, se les aplicó el cuestionario, con ayuda de una herramienta en línea, para que evaluaran el tutorial de APA.

\section{Selección de $O A$}

En la programación didáctica del curso, mencionado más arriba, una de las unidades didácticas más relevantes es la de estilos bibliográficos, razón por la que al seleccionar el pre-testado del cuestionario se optó por un OA que apoyase su aprendizaje.

A estos efectos, así como en consideración de los aspectos incluidos en los criterios evaluativos apuntados, se seleccionó un banco de OA destinados a programas de alfabetización en información, el Information Literacy Resource Bank de la Cardiff University: https://ilrb.cf.ac.uk/citingreferences/apatutorial/index.html. La consulta de OA en este banco permitió la selección de la Evaluación del APA Referencing Tutorial.

La selección de este OA se debe a que: a) es un recurso que apoya significativamente el aprendizaje de un estilo bibliográfico esencial para los estudiantes en las áreas de ciencias sociales; b) está desarrollado y reside en un banco de recursos diseñado específicamente para apoyar la alfabetización en información; c) reúne muchas de las características que requiere un $\mathrm{OA}$, lo que facilita la revisión con base en los criterios elegidos para ese fin. Otra importante razón de esta selección es la propia experiencia que tienen los autores en cuanto a ejercicios competenciales, por cuanto los alumnos preparan sus citas y referencias en Microsoft Word de forma "manual", sin ayuda del gestor bibliográfico (RefWorks), y posteriormente lo hacen con apoyo del gestor. El sitio es la versión electrónica de una guía impresa de la editorial: http://bcs.bedfordstmartins.com/resdoc5e/RES5e_ch09_o.html

\section{Análisis de resultados y discusión}

Considerando los criterios evaluativos expuestos en la Tabla 1, se definió un cuestionario de 15 preguntas de la evaluación que fue publicado en línea en Google Docs, lo que permitía recibir los resultados al día y cuya dirección es https://docs.google.com/forms/d/17oCAxk0OcvNuDdFEVd7Pp1cJCmJmn 6yzABBNBHsaJhM/viewform

La mecánica del cuestionario del pre-testado era: 


\section{INSTRUCCIONES}

Ingrese al siguiente sitio, resuelva el tutorial y responda a la siguiente evaluación: https://ilrb.cf.ac.uk/citingreferences/apatutorial/index.html

Tabla 2. Preguntas constituyentes del cuestionario pre-testado.

\begin{tabular}{|l|}
\hline $\begin{array}{l}\text { Nombre: } \\
\text { Programa de estudios: }\end{array}$ \\
\hline 1. Identifique el repositorio en el que está alojado el recurso. \\
\hline 2. ¿Considera que el sistema se ejecuta de forma rápida? Sí No \\
\hline 3. ¿Requirió algún programa o aplicación específica adicional para la ejecución del sistema? Sí No \\
\hline 4. ¿Encontró algún problema relacionado con el funcionamiento y vigencia de los hipervínculos? Sí No \\
\hline 5. ¿Considera que los elementos que conforman el sistema tienen un propósito educativo? Sí No \\
\hline 6. ¿Considera que las actividades que incluye el sistema son suficientes para cumplir los objetivos de \\
aprendizaje? Sí No \\
\hline 7. ¿Considera que es necesario un entrenamiento previo para el uso del sistema? Sí No \\
\hline 8. ¿El sistema resultó amigable en su forma de uso? Sí No \\
\hline 9. ¿Considera que la información que aparece en el recurso es vigente? Sí No \\
\hline 10. ¿Encontró algún error en el recurso? Sí No \\
\hline $\begin{array}{l}\text { 11. Después de concluir el ejercicio ¿cuál considera que es el objetivo que mejor se cumple de los que fueron } \\
\text { definidos al inicio del recurso (Aims of this Tutorial)? }\end{array}$ \\
\hline $\begin{array}{l}\text { 12. ¿Encontró algún elemento estimulante para el autoaprendizaje de los contenidos del recurso? Si así fue } \\
\text { ¿Cuál es ese elemento? }\end{array}$ \\
\hline $\begin{array}{l}\text { 13. ¿En qué medida la herramienta de autoevaluación le ayudó a reforzar los contenidos? } \\
\text { Mucho } 12345 \text { Nada }\end{array}$ \\
\hline 14. ¿ildentificó usted los permisos para el uso del recurso? Sí No \\
\hline 15. ¿identificó usted los derechos de autor en el recurso? Sí No \\
\hline
\end{tabular}

Fuente: elaboración propia

Las respuestas resultantes han arrojado los siguientes resultados:

1. E1 $7 \%$ de los participantes pudo identificar con claridad el repositorio en el que está alojado el recurso (accesibilidad).

2. El $100 \%$ considera que el sistema se ejecuta con rapidez (accesibilidad).

3. Aunque en apariencia no es necesario, el $7 \%$ de las personas consideró necesario instalar algún programa o aplicación específica para utilizar el tutorial (accesibilidad).

4. Sólo el 7 \% de los participantes refiere que encontró algún problema relacionado con el funcionamiento y vigencia de los hipervínculos (durabilidad).

5. El $100 \%$ considera que el sistema tiene un propósito educativo (diseño instructivo).

6. El $14 \%$ de las personas considera que las actividades que incluye el tutorial son insuficientes para cumplir los objetivos de aprendizaje (interactividad). 
7. Resulta interesante encontrar que $43 \%$ de los participantes consideró que es necesario un entrenamiento previo para usar el sistema (diseño instructivo).

8. A pesar de los resultados de la pregunta anterior, el $100 \%$ consideró que el sistema es amigable en su uso (usabilidad).

9. El $100 \%$ de los participantes consideró que la información que contiene el recurso es vigente (durabilidad).

10. Solamente el $7 \%$ de las personas encontró algún error en el tutorial (interactividad).

11. La mayoría de las personas consideró que el objetivo del tutorial que mejor se cumplió es el relacionado con la forma en cómo citar documentos con el estilo APA (How to cite sources in your written work using the APA referencing style) (diseño instructivo).

12. El $86 \%$ sí encontró algún elemento estimulante para el autoaprendizaje de los contenidos del recurso; sin embargo, el 7 \% lo consideró básico, en tanto que otro 7 \% manifestó que no encontró ninguno (diseño instructivo).

13. El $93 \%$ de los participantes consideró que la herramienta de autoevaluación les ayudó a reforzar los contenidos, valorando este aspecto con puntuaciones de 4 y 5, en una escala del 1 al 5 . Únicamente el 7 \% otorgó la valoración más baja a la herramienta de autoevaluación (diseño instructivo).

14. La identificación de los permisos quedó muy dividida entre las personas: el $57 \%$ pudo determinar cuáles eran, en tanto que el $43 \%$ no lo pudo hacer (autoridad).

15. El $29 \%$ de las personas no pudo identificar la información relacionada con los derechos de autor del tutorial (autoridad).

El análisis diagnóstico de estos resultados permite ciertas premisas de trabajo para su discusión, bastante interesantes, teniendo muy presente que en este pre-testado no se trataba de evaluar el OA (esto será en una fase posterior, dentro de un programa competencial bien definido), sino la validez del cuestionario para discentes (evaluadores "inducidos" del OA) y docentes (evaluadores de las competencias del discente cuando evalúan un OA). De este modo, podemos categorizar los resultados:

a) Perspectiva del docente. La incorporación de los OA a un programa educativo competencial muestra como evidencias útiles las siguientes:

- El uso de OA contextualiza con eficacia al alumno dentro de un entorno educativo y de aprendizaje (un 100 \% de la pregunta 5). Esto 
es del mayor interés por cuanto el alumno está predispuesto al uso del OA no como fuente de información y resultado de búsquedas para un trabajo académico, sino como un recurso educativo digital que le proporcionará "resultados de aprendizaje".

- Hacer girar a un programa educativo competencial sobre un OA permite que los alumnos tomen conciencia cierta sobre su necesidad de superar una simple formación en recursos informativos (más de un $40 \%$, en la pregunta 7), por la asimilación de que el aprendizaje óptimo basado en las potencialidades del OA depende de adquirir, mediante una "alfabetización", las competencias para una mejor obtención de conocimiento. Sin duda, los programas de alfabetización en información cobran mayor sentido en ámbitos educativos y más en Educación Superior.

- Los OA se manifiestan como un instrumento sumamente útil para el fomento del aprendizaje autónomo en programas educativos competenciales, lo que es el fundamento para el aprendizaje permanente: así se acredita en la muy positiva valoración en torno a la satisfacción con las actividades propuestas; esto es, la visible interactividad del recurso (86 \% de la pregunta 6), cómo estimula el autoaprendizaje ( $86 \%$ de la pregunta 12 ), pero especialmente cuál es la utilidad de la autoevaluación en el OA (93\% de la pregunta 13).

- El diseño tecnológico e instruccional de los OA deja en evidencia una constatación de primer orden. Los alumnos, procedentes de un entorno que ya los reconoce como nativos digitales, no parecen tener mayores problemas en acceder al recurso y manejarlo tecnológicamente: ésta es la demostración de las respuestas 2 (100 \% sobre la rapidez de respuesta a partir del OA), 4 (93\% no tuvieron problemas con hipervínculos), 8 (100\% declaró amigabilidad del OA), 9 y 10 (100\% aprobó la vigencia de los contenidos, $93 \%$ no tuvo errores, respuestas que inducen a evaluar el conocimiento experto de navegación del alumno dentro del OA más que la pericia en evaluar la calidad del contenido). Además, los alumnos encuentran mayores dificultades en el "uso" de los contenidos, ésos que deberá procesar para obtener conocimiento, según apuntan las respuestas sumamente significativas a la pregunta 1 (el $93 \%$ no identificó el repositorio que acoge al OA), 14 (un $43 \%$ no identificó los permisos en el OA), 15 (casi un tercio no visualizó los derechos de autor).

b) Perspectiva del discente. Los OA parecen tener un efecto decididamente positivo entre los alumnos, desde varios puntos de vista: 
- Todo programa educativo competencial tiene como requisito previo, imprescindible y básico el reconocimiento, por parte del educando, de sus propias deficiencias en competencias en información, algo complicado entre alumnos pertenecientes a generaciones de nativos digitales. La estructura tecnológica e instruccional de los OA es en extremo útil para este objetivo educativo fundamental: evidencia que la pericia tecnológica (remitimos a las ratios ya apuntadas más arriba en las preguntas 2, 4, 8, 9 y 10) no implica una pericia en la transferencia de información a conocimiento (de nuevo remitimos a las ratios de las preguntas 1, 13, 14 y 15). Esto nos demuestra la necesidad de que el programa educativo competencial se articule en torno al concepto de las CI2 (competencias informáticas e informacionales): las competencias informáticas son condición necesaria pero no suficiente para el conocimiento en la Web, pero sin ellas no se puede acceder a las competencias en información. Este complemento es una enseñanza sumamente útil para los docentes: los resultados de las preguntas 5 y 7 son, en principio, una muestra de por dónde definir una estrategia.

- El uso didáctico y educativo de los OA debe hacerse dentro de un programa didáctico planificado y una estrategia educativa programada, ya que los alumnos manifiestan una visión del OA reduccionista y muy unilateral: el $93 \%$ no ve necesario instalar nuevas aplicaciones para el uso del OA (pregunta 3), la mayoría de los alumnos identificó la utilidad del OA para un objetivo concreto, cómo citar (pregunta 11). Desde esta perspectiva, el uso de los OA como simple material didáctico de apoyo estaría restando su enorme potencialidad educativa derivada de su asociatividad de contenidos, interoperabilidad, multisecuencialidad, reutilización y versatilidad en contextos educativos.

- Los OA tienen una efectividad educativa tanto mayor cuanto más aptos son los educandos en la Web 2.0: la interactividad es uno de los aspectos que más y mejor valoran los alumnos en los docentes. Es el uso interactivo de sus contenidos lo que hace que los alumnos conecten a los OA con el autoaprendizaje, el aprendizaje significativo y el aprendizaje permanente, conforme acreditan las ratios de las preguntas 6, 12 y 13. De este modo, los OA demuestran ser el instrumento idóneo para uno de los objetivos educativos fundamentales de un programa de alfabetización en información: la actualización autónoma de conocimientos por la posesión de competencias en información. 
c) Perspectiva del cuestionario. No debemos olvidar que estamos presentando la propuesta de un cuestionario, para el que se ha efectuado un pre-testado. En este orden de cosas, se hace necesario un doble análisis:

- La selección del OA parece acertada a partir de los resultados respecto de un pre-testado, especialmente tomando en cuenta las necesidades de estudiantes de Posgrado en Ciencias Sociales. Sin embargo, la selección adolece de algunas propiedades que demuestran hasta qué punto son básicos los OA en programas competenciales: a) está demasiado próximo a un tutorial; b) es en exceso "instrumentalista" para un propósito educativo muy concreto (citar); c) no contempla algunas características propias de los OA más relevantes en los ámbitos educativos competenciales.

- La formulación de las preguntas demuestra ser eficaz, pues son directas respecto al objeto cuestionado, lo que ha permitido: a) la perfecta comprensión de lo que se pedía, por parte del encuestado; y b) se ha evitado la obtención de respuestas "inducidas" por una interrogación condicionante. Sin embargo, la utilidad del cuestionario para un programa educativo competencial muestra una vía perfectible, a partir de este pre-testado: a) las preguntas son muy específicas en el descubrimiento de aspectos concretos en el uso del OA, por lo que la extrapolación de resultados hacia aspectos educativos se hace compleja; b) deben incorporarse preguntas que permitan al educando una mayor "evaluación" del OA respecto a sus contenidos, multimedialidad, interoperabilidad, reutilización, usabilidad y diseño instructivo, aspectos que no han podido ser cuestionados por la propia naturaleza del OA seleccionado.

\section{Conclusiones}

El desarrollo del presente trabajo de investigación nos lleva a concluir cuatro fenómenos muy significativos:

1. La necesidad de que en los programas educativos de alfabetización en información se haga una definitiva, complementaria y sumamente valiosa complementariedad entre la competencia en información, o las CI2, con el comportamiento informacional, un aspecto que desborda conceptual y metodológicamente una aproximación al estudio de usuarios y el consumo de información científica. El comportamiento informacional nos re- 
mite de forma directa a la actitud y conducta del educando, fundamento imprescindible de las "competencias" en cualquier ámbito.

2. La definición de grupos-meta de estudio, su tipificación por consumo y comportamiento informacionales, no deben hacernos perder de vista que la eficacia de los programas competenciales de alfabetización en información, radica fundamentalmente en determinar la finalidad competencial según el grupo, así como el "espacio" o ambiente formativo; esto es, los programas de alfabetización en información deben ser modulares, escalables y flexibles, especialmente útiles en bibliotecas digitales con vocación formativa. Esta circunstancia es la que hace a los OA especialmente útiles en estos programas.

3. Una aproximación analítica a los programas de alfabetización en información existentes demuestra la variedad de materiales digitales didácticos utilizados, lo que hace particularmente evanescente su proyección educativa. Si se ha hecho un importante esfuerzo conceptual por definir la alfabetización en información o el desarrollo de competencias en información, digital fluency y CI2, parece imprescindible llegar a ciertas convenciones respecto a los contenidos digitales específicos para estos programas. La argumentación de este trabajo parece demostrar que, conforme a los objetivos competenciales de la alfabetización en información, los ODE y OA deben ser los genuinos materiales didácticos.

4. La selección y evaluación de contenidos digitales, atendiendo a un dominio experto de los métodos del aprendizaje colaborativo, los enlaces en redes, los conectores del discurso electrónico y la gestión de contenidos web, son el nudo competencial en un programa de alfabetización en información, por lo que están surgiendo importantes iniciativas en la medición del impacto de apropiación informática e informacional, lo que implica desarrollar instrumentos de selección y evaluación que les sean propios, naturalmente también para los $\mathrm{OA}$, como material idóneo de estos programas. Esto plantea la necesidad imperiosa de determinar el instrumento evaluativo idóneo, lo que reclama una precisión conceptual y metodológica: a) encuesta evaluativa, como un repertorio de preguntas diseñadas conforme a los métodos estadísticos, sin duda, pero que midan (cuantitativa o cualitativamente) la opinión, percepción de fenómenos o valoración de caracteres sobre un tópico determinado, un instrumento para destrezas que se apoye en una checklist de los aspectos y un documento sobre los criterios; b) cuestionarios, instrumento evaluativo sólido en su uso para medir las habilidades, cuya interpretación de los datos se hace en estrecha relación con un conjunto de indicadores, basados en estándares definidos y en categorías evaluativas de interpretación; c) indi- 
cadores, definidos por la ONU en Integrated and coordinated implementation and follow-up of major United Nations conferences and Summits como "herramientas para clarificar y definir, de forma más precisa, objetivos e impactos [...] son medidas verificables de cambio o resultado [...] diseñadas para contar con un estándar contra cual evaluar, estimar o demostrar el progreso [...] con respecto a metas establecidas, facilitan el reparto de insumos, produciendo [...] productos y alcanzando objetivos" (United Nations Statistics Division, 1999: s. pág.), por lo que deberán entenderse ahora como el elemento guía del instrumento evaluativo, que se proyecten en plantillas de criterios evaluables, se formulen estadísticamente y se nutran de cuestionarios, cuyas preguntas evaluadoras se relacionarán directamente con su indicador para interpretación. En este sentido se ha orientado el esfuerzo investigador del trabajo.

\section{RefERENCIAS}

Barritt, C. y Alderman, F. L. (2004), Creating a reusable learning objects strategy: Leveraging information and learning in a knowledge economy, San Francisco, CA: Pfeiffer.

Barry, C. A. (1997), "Information skills for an electronic world: Training doctoral research students", en Journal of Information Science, 23 (3), pp. 225-238. DOI: 10.1177/016555159702300306

Bueno, G. (2010), Modelo de repositorio institucional de contenido educativo (RICE): la gestión de materiales digitales de docencia y aprendizaje en la biblioteca universitaria, tesis doctoral, Getafe, eArchivo Universidad Carlos III de Madrid. Disponible en: http://e-archivo. uc3m.es/handle/10016/9154 [Fecha de consulta: 11 de febrero de 2013].

Calzada Prado, J. (2010), Repositorios, bibliotecas digitales y CRAI: los objetos de aprendizaje en la educación superior, Buenos Aires: Alfagrama.

Catalano, A. (2010), "Using ACRL standards to assess the information literacy of graduate students in an education program", en Evidence Based Library and Information Practice, 5 (4), pp. 21-38.

Consortium of University Research Libraries \& Research Information Network (2007), Researchers' use of academic libraries and their services: A report, London: Research Information Network [and] Consortium of University Research Libraries (CURL).

Dewald, N.; Scholz-Crane, A.; Booth, A. y Levine, C. (2000), "Information literacy at a distance: Instructional design issues", en The Journal of Academic Librarianship, 26 (1), pp. 33-44. 
Doorten, M.; Giesbers, B.; Janssen, J.; Daniels, J. y Koper, R. (2004), "Transforming existing content into reusable learning objects", en R. McGreal (ed.), Online education using learning objects, London: Falmer Press, pp. 116-127.

George, C.; Bright, A.; Hurlbert, T.; Linke, E. C.; St. Clair, G. y Stein, J. (2006), "Scholarly use of information: Graduate students' information seeking behavior”, en Information Research, 11 (4), pp. 11-14.

Gértrudix, M.; Álvarez, S.; Galisteo, A.; Gálvez, M. D. C. y Gértrudix, F. (2007), "Acciones de diseño y desarrollo de objetos educativos digitales: programas institucionales", en RUSC. Revista de Universidad y Sociedad del Conocimiento, 4 (1), pp. 14-25.

Harrington, M. R. (2009), "Information literacy and research-intensive graduate students: Enhancing the role of research librarians", en Behavioral E Social Sciences Librarian, 28 (4), pp. 179-201.

Hernández, Y.; Silva, A. y Velásquez, C. (2012), "Instrumento de Evaluación para Determinar la Calidad de los Objetos de Aprendizaje Combinados Abiertos de tipo Práctica", en LACLO, 3 (1). Disponible en: http://laclo.org/papers/index.php/laclo/article/view/20 [Fecha de consulta: 5 de marzo de 2013].

Hodgins, H. W. (2002), “The Future of Learning Objects”, en e-Technologies in Engineering Education: Learning Outcomes Providing Future Possibilities, Jack R. Lohmann, Georgia Institute of Technology, USA; Michael L. Corradini, University of Wisconsin-Madison, USA (eds.), ECI Symposium Series, Volume P01. Disponible en: http://dc.engconfintl.org/etechnologies/11 [Fecha de consulta: 13 de marzo de 2013].

Hoffmann, K.; Antwi-Nsiah, F.; Feng, V. y Stanley, M. (2008), "Library research skills: A needs assessment for graduate student workshops", en Issues in Science and Technology Librarianship, núm. 53. Disponible en: http://www.istl.org/08-winter/refereed1. html [Fecha de consulta: 15 de noviembre de 2012].

Holmes, J. (2003), "Online learning objects: Helping faculty teach information literacy (and more)”, Public Services Quarterly, 1 (4), pp. 1-9.

Kayongo, J. y Helm, C. (2010), "Graduate students and the library: A survey of research practices and library use at the university of notre dame”, en Reference \& User Services Quarterly, 49 (4), pp. 341-349.

Kohl-Frey, O. (2008), "Information literacy for advanced users: A german perspective”, en Public Services Quarterly, 3 (3), pp. 71-93.

Koper, R. (2003), "Combining reusable learning resources and services with pedagogical purposeful units of learning”, en A. Littlejohn (ed.), Reusing Online Resources, London: Kogan Page, pp. 46-59.

LTSC (Learning Technology Standards Committee) (2002), Draft Standard for Learning Object Metadata: IEEE Standard 1484.12.1, Nueva York: Institute of Electrical and Electronics Engineers. Disponible en: http://129.115.100.158/txlor/docs/IEEE_LOM_ 1484_12_1_v1_Final_Draft.pdf 
Martínez Naharro, S. et al. (2007), "Los objetos de aprendizaje como recurso de calidad para la docencia: criterios de validación de objetos en la Universidad Politécnica de Valencia”, en Actas del IV Simposio Pluridisciplinar sobre Diseño, Evaluación y Desarrollo de Contenidos Educativos Reutilizables, 19, 20 y 21 de septiembre 2007, Bilbao: Universidad del País Vasco. Disponible en http:// spdece07.ehu.es/actas/Naharro.pdf [Fecha de consulta: 4 de marzo de 2013].

Marzal, M. A.; Calzada, F. J. y Vianello, M. (2008), "Criterios para la evaluación de la usabilidad de los recursos educativos virtuales: un análisis desde la alfabetización en información”, en Information Research, 13 (4). Disponible en: http://informationr.net/ ir/13-4/infres134.html [Fecha de consulta: 6 de marzo de 2013].

Marzal, M. A. y Parra, P. (2010), "Bibliotecas integradas: alfabetización en información como estímulo de cooperación”, en El Profesional de la Información, 19 (5), septiembre-octubre, pp. 463-468.

Marzal, M. A.; Parra, P. y Colmenero, M. J. (2011), "La medición de impacto y evaluación de programas de alfabetización en información para bibliotecas escolares", en Revista Española de Documentación Científica, 34 (2), pp. 190-211.

McGreal, R. (2004), "Learning Objects: A Practical Definition.” en International Journal of Instructional Technology and Distance Learning, 1 (9), pp. 21-32. Disponible en: http://www.itdl.org/ journal/sep_04/article02.htm

McGreal, R. (2008), "A typology of learning object repositories", en Heimo H. Adelsberger et al., Handbook on Information Technologies for Education and Training, Nueva York, NY: Springer.

Merrill, M. D. (1983), "Component display theory", en C. Reigeluth (ed.), Instructional design theories and models, Hillsdale, NJ: Erlbaum Associates.

Mestre, L. S. (2010), "Matching Up Learning Styles with Learning Objects: What's Effective?”, en Journal of Library Administration, 50 (7-8), pp. 808-829.

Mestre et al. (2011), "Learning objects as tools for teaching information literacy online: A survey of librarian usage", College $E$ Research Libraries, 72 (3), p. 236.

Metros, S. E. (2005), "Learning objects: A rose by any other name”, en Educause Review, 40 (4), pp. 12-13.

Metros, S. E. y Bennett, K. (2002), "Learning objects in higher education”, en Educause Research Bulletin, 19, pp. 1-10. Disponible en: https://net.educause.edu/ir/library/pdf/ERB0219.pdf

Moreno, F. y Bailly-Baillière, M. (2002), Diseño instructivo de la formación on-line: Aproximación metodológica a la elaboración de contenidos, Barcelona: Ariel.

Newby, J. (2011), “Entering unfamiliar territory: Building an information literacy course for graduate students in interdisciplinary areas", en Reference \& User Services Quarterly, 50 (3), pp. 224-229. 
Pinto, M.; Gómez, C. y Fernández, A. (2012), "Los recursos educativos electrónicos: perspectivas y herramientas de evaluación”, en Perspectivas em Ciência da Informaçao, 17 (3), pp.82-99.

Polsani, P. R. (2003), "Use and abuse of reusable learning objects", en Texas Digital Library, 3 (4). Disponible en: https://journals.tdl.org/ jodi/index.php/jodi/article/view/89/88 [Fecha de consulta: 13 de marzo de 2013].

Romanos de Tiratel, S. (2000), "Accessing information use by humanists and social scientists: a study at the Universidad de Buenos Aires, Argentina", en The journal of academic librarianship, 26 (5), pp. 346-354.

Sadler, E. y Given, L. M. (2007), "Affordance theory: A framework for graduate students' information behavior", en Journal of Documentation, 63 (1), pp. 115-141. DOI:10.1108/00220410710723911

Saiti, A. y Prokopiadou, G. (2008), "Post-graduate students and learning environments: Users' perceptions regarding the choice of information sources", en The International Information $\& \mathrm{Li}$ brary Review, 40 (2), pp. 94-103.

Somoza, M. y Fernández, C. (2011), “Tutoriales web: indicadores y ejemplos de buenas prácticas", en El Profesional de la Información, 20 (1), pp. 38-46.

Tarango, J. y Marzal, M. A. (2011), "Funcionalidad de la alfabetización informativa y sus ámbitos de actuación: aproximaciones a los entornos mexicano y español", en Revista Interamericana de Bibliotecología, 34 (3), pp. 283-299.

United Nations Statistics Division (1999), Integrated and coordinated implementation and follow-up of major United Nations conferences and summits at all levels, Nueva York, NY: United Nations. Disponible en: http://www.un.org/documents/ecosoc/docs/1999/e19 99-11

Viñas, V. (2004), "Evaluación cualitativa de programas de desarrollo regional en zonas rurales”, en Revista de Estudios Regionales, 71, pp. 13-36.

Wiley, D. A. (2000), Connecting learning objects to instructional design theory: a definition, a metaphor, and a taxonomy, Utah State University. 